\title{
Relative and Absolute Risk of Tendon Rupture with Fluoroquinolone and Concomitant Fluoroquinolone/Corticosteroid Therapy: Population-Based Nested Case-Control Study
}

\author{
Daniel R Morales $^{1}$ (D) Jim Slattery ${ }^{2} \cdot$ Alexandra Pacurariu $^{2} \cdot$ Luis Pinheiro $^{2} \cdot$ Patricia McGettigan $^{2,3} \cdot$ Xavier Kurz $^{2}$
}

Published online: 21 November 2018

(c) The Author(s) 2018, corrected publication 2019

\begin{abstract}
Background and Objective Tendon rupture can result from fluoroquinolone exposure. The objective of this study was to quantify relative and absolute risk and determine how risk is affected by timing of exposure.

Methods The UK Health Improvement Network primary care database was used to perform a nested case-control study measuring the association between fluoroquinolone exposure and tendon rupture. Adults with tendon rupture were matched on age, sex, general practice and calendar time to four controls selected from a cohort prescribed systemic fluoroquinolone or co-amoxiclav antibiotics. The relative and absolute risk of tendon rupture with fluoroquinolone exposure was calculated. Results Current fluoroquinolone exposure was associated with an increased risk of any tendon rupture (adjusted incidence rate ratio [aIRR] 1.61, 95\% CI 1.25-2.09) and Achilles tendon rupture (aIRR 3.14, 95\% CI 2.11-4.65) that persisted for 60 days. Risk increased with cumulative exposure and was greatest when co-prescribed with oral corticosteroids (aIRR 19.36, 95\% CI 7.78-48.19 for Achilles tendon rupture). The adjusted rate difference (aRD) with fluoroquinolone exposure was 2.9 and 2.1 per 10,000 patients for any and Achilles tendon rupture, respectively, and was greatest in people aged $\geq 60$ years prescribed concomitant oral corticosteroid therapy (aDR 19.6 for any tendon and 6.6 Achilles tendon rupture per 10,000). No association was seen with co-amoxiclav or statin exposure, or with biceps or other tendon ruptures.

Conclusions Risk of tendon rupture with fluoroquinolones depends on timing, cumulative dose and concomitant exposure to oral corticosteroids. Absolute risk significantly varied by age and concomitant corticosteroid exposure, affecting elderly patients the greatest.
\end{abstract}

Electronic supplementary material The online version of this article (https://doi.org/10.1007/s40261-018-0729-y) contains supplementary material, which is available to authorized users.

Daniel R Morales

d.r.z.morales@dundee.ac.uk;

Daniel.Morales@ext.ema.europa.eu

1 Division of Population Health and Genomics, School of Medicine, University of Dundee, Mackenzie Building, Kirsty Semple Way, Dundee DD2 4BF, UK

2 Pharmacovigilance and Epidemiology Department, European Medicines Agency, London, UK

3 William Harvey Research Institute, Queen Mary University of London, London, UK

\section{Key Points}

Risk of tendon rupture with fluoroquinolones depends upon timing, cumulative dose and concomitant exposure to oral corticosteroids.

Absolute risk varied by age and concomitant corticosteroid exposure, affecting elderly patients the greatest.

\section{Introduction}

Fluoroquinolone antibiotics are widely used to treat a broad range of infections, including those of the urinary respiratory and gastrointestinal systems, with variation in the prevalence of prescribing and indications for use within different health systems [1]. However, fluoroquinolones cause important side effects as listed in their Summary of Product Characteristics 
(SPC), which include tendon ruptures that are listed as occurring very rarely with a frequency of less than 1 per 10,000 patients $[2,3]$. Tendon ruptures manifest as complete or partial rupture of the tendon and are an important cause of morbidity, affecting both functional ability and quality of life with treatment often involving invasive surgery $[4,5]$. The incidence of tendon rupture in the general population varies according to tendon site, with estimates ranging from 4.7 to 55.2 per 100,000 person-years for Achilles tendon rupture, and 2.6 to 5.4 per 100,000 person-years for biceps tendon rupture [6-8]. The mechanism for fluoroquinoloneinduced tendon rupture remains uncertain, but has been linked to changes in collagen fibrils following alterations in the regulation of matric metalloproteinases associated with non-traumatic rupture [9]. Although some risk factors for tendon rupture have been identified such as increasing age, male gender, obesity, diabetes, corticosteroid therapy and recreational sports [10-12], a further understanding of risk factors is needed to identify at-risk individuals and minimize unintended harm.

In 1995, the US Food and Drug Administration (FDA) updated fluoroquinolone product labelling with a warning about the possibility of tendon rupture, followed by a 'black box' warning in 2008 [13]. In May 2016, the FDA conducted a review of disabling and potentially irreversible serious side effects of fluoroquinolones resulting in a restriction of their use in less severe infections of acute sinusitis, acute bronchitis and uncomplicated urinary tract infections when other treatment options are available [14]. The European Medicines Agency (EMA) Pharmacovigilance Risk Assessment Committee (PRAC) has also recently reviewed the persistence of fluoroquinolones adverse reactions to help determine the need for any restriction in indication, and required further data to characterise the risk of such reactions [15]. Whilst the risk of tendon rupture is recognized, there is inconsistency in its reported size with relative risk ranging between 1.9- and 5.3-fold increased risk, with limited information on absolute risk [10,16-21]. The aim of this study was to quantify the relative and absolute risk of tendon rupture with fluoroquinolone exposure, and to investigate factors influencing this risk.

\section{Methods}

\subsection{Data Source}

The THIN database contains electronic patient records extracted from $>500$ general practices across the UK covering approximately $6 \%$ of the UK population. Data are representative of the UK population in terms of age, gender, deprivation status and geographical distribution [22]. Data are linked via an anonymous ID number allowing patients to be followed longitudinally over time. THIN contains diagnostic, prescription and lifestyle information. Diagnoses, symptoms, procedures and other relevant health information are coded using the Read Code clinical classification system. The Read Code system has been the standard approach to recording patient data in UK primary care since 1990, and is a hierarchical classification system, linked to the International Classification of Diseases [23]. Data quality control measures available in THIN include The Acceptable Mortality Reporting (AMR) data specific to each practice and defines the date from which computerised recording of mortality data has reached an acceptable standard [24].

\subsection{Study Population}

The study population consisted of adults $\geq 18$ years of age identified in THIN between 1 January 1999 and 31 December 2015 who were issued at least one prescription of coamoxiclav or fluoroquinolone antibiotic with a systemic route of administration. Co-amoxiclav was chosen so that controls were sampled from a more representative population prescribed antibiotics to circumvent problems related to confounding by indication and by severity. Cohort entry was defined as the date of the first co-amoxiclav or fluoroquinolone prescription for systemic administration after the latest of the following criteria: start of the study period (1 January 1999); the practice's AMR date; the patient's 18th birthday; date of registration with a general practice +1 year. In this regard, all participants were required to have at least 1 year of observation prior to cohort entry. Cohort exit was defined by the earliest of the following criteria: occurrence of the outcome; deregistration from the general practice; death; date of last data collection from the general practice; end of the study period (31 December 2015).

\subsection{Study Design and Outcomes}

A nested case-control study was used to efficiently account for time-varying confounders and time-varying exposure to prescribed medicines [25]. The date of the first event occurring after cohort entry was the index date for case subjects. Tendon ruptures were first evaluated as any tendon ruptures and were then grouped according to their incidence: Achilles tendon; biceps tendon; and other tendon ruptures (consisting of triceps, hand/wrist, hamstrings/quadriceps, foot, shoulder, and non-specifically coded tendon ruptures) (see Online Supplementary Table S1)

\subsection{Control Selection}

Up to four controls were randomly selected and matched to each case on age decile, gender, general practice and calendar year of cohort entry using incidence density sampling 
[25]. The risk-set date for controls was the index date for cases. With incidence density sampling, 'controls' are a selection of person-moments from individuals who have not experienced the event at the index date. In this regard, controls may be selected more than once, and people who subsequently become cases may be selected as controls at earlier time points. Eighty-two cases of tendon rupture (1.7\%) were unmatched to controls following the first round of matching criteria, and were subsequently included matched on age decile, gender and calendar year of cohort entry only, with sensitivity analyses conducted excluding these risk sets from the analysis.

\subsection{Exposures}

Systemic fluoroquinolone (ciprofloxacin, moxifloxacin, levofloxacin, norfloxacin and ofloxacin) and co-amoxiclav antibiotic exposure was measured by identifying prescriptions issued within a pre-specified risk window prior to the index date. Fluoroquinolone and co-amoxiclav exposure was primarily defined as current exposure when one or more prescriptions were issued in a 30-day exposure risk window prior to the index date. Exposure risk windows 31-60 days, 61-90 days and 91-180 days prior to the index date were then examined for systemic fluoroquinolone exposure to assess past exposure. Cumulative fluoroquinolone and coamoxiclav exposure was measured as the total number of days of systemic fluoroquinolone or systemic co-amoxiclav exposure prescribed within each risk window. Cumulative days exposure was calculated by dividing the prescription quantity information by the standard administration schedule for each type of antibiotic as follows: oral co-amoxiclav three times a days; oral levofloxacin, moxifloxacin and norfloxacin once a day; ciprofloxacin and ofloxacin twice a day [26]. Prescriptions for oral suspension formulations were similarly calculated taking into account quantity information recorded in millilitres. Cumulative exposure was examined as a continuous variable predicting the increased risk associated with each additional day of therapy during the risk window and also modelled with a categorical variable using the median days of exposure and interquartile range.

\subsection{Confounders}

Analyses were adjusted for exact age, sex and practice-level socioeconomic deprivation (inherent in the matching criteria), smoking status, body mass index (BMI), exposure to oral and injectable corticosteroid therapy and statin therapy (defined as prescriptions issued within 90 days of the index date), and prior history of tendon rupture. Co-morbidity was accounted by adjusting for a history of hypothyroidism, hyperparathyroidism and the Charlson co-morbidity index, and then by modelling individual conditions in the Charlson co-morbidity index as a sensitivity analysis. The Charlson co-morbidity index is defined using the following clinical conditions: myocardial infarction, congestive heart failure, peripheral vascular disease, dementia, cerebrovascular disease, chronic lung disease, connective tissue disease, peptic ulcer, chronic liver disease, diabetes, hemiplegia, moderate or severe kidney disease, diabetes with complications, liver disease, cancer, cancer with metastasis and AIDS [27].

\subsection{Data Analysis}

Conditional logistic regression was used to calculate odds ratios for the association between tendon rupture and fluoroquinolone exposure and co-amoxiclav exposure. Using an incidence density sampling approach, odds ratios calculated in this way are estimators of incidence rate ratios (IRRs). Interaction between fluoroquinolone exposure and oral corticosteroid exposure was tested on the multiplicative scale. This was not done for statins as there was no statistically significant independent association with exposure. Adjusted rate differences (per 10,000 per year) were calculated for significant associations providing an absolute measure of effect [28]. Risk of exposure was presented for any tendon rupture and by type of tendon rupture.

Multiple imputation was used to impute missing data on body mass index (9.5\% missing) and smoking status $(2.1 \%$ missing). The imputation model included all variables relating to clinical characteristics, outcomes, medications, and fluoroquinolone and co-amoxiclav exposure. Multiple imputation used fully conditional specification, with linear regression for continuous variables (BMI) and logistic regression for categorical variables (smoking status) with five imputations and analysed using Rubin's rules [29]. Sensitivity analyses were conducted varying the exposure risk window duration to 60 and 90 days, modelling individual Charlson co-morbidities, excluding traumatic tendon ruptures, excluding people with prior history of tendon rupture, and undertaking a complete case analysis. Analysis was carried out using SAS Enterprise Guidev7.1 and STATAv15. The study was approved by the THIN Scientific Review Committee (17THIN087).

\section{Results}

The cohort contained 1,351,780 adult patients prescribed $\geq 1$ prescription for fluoroquinolone $(34.3 \%)$ or co-amoxiclav $(65.7 \%)$ antibiotics (mean age of cohort 52.8 years, $57.0 \%$ female). Overall, 4836 tendon rupture events were identified during cohort follow-up (incidence of any tendon rupture 5.9 per 10,000 person-years (pyrs) and of Achilles tendon rupture 1.9 per 10,000 pyrs) that were matched to 18,356 controls (Table 1). Cases and controls 
Table 1 Characteristics of matched cases and controls

\begin{tabular}{lll}
\hline Characteristic & Cases & Controls \\
\hline Number of individuals & 4836 & 18,356 \\
Female sex, no. (\%) & $1876(38.8)$ & $7164(39.0)$ \\
Age (years), mean \pm SD & $61.6(15.5)$ & $61.3(15.3)$ \\
Years of follow-up, mean \pm SD & $5.0(3.7)$ & $5.0(3.7)$ \\
Body mass index, mean \pm SD & $28.1(5.9)$ & $27.7(5.7)$ \\
Charlson co-morbidity index, mean \pm SD & $1.32(1.61)$ & $1.27(1.64)$ \\
Hypothyroidism, no. (\%) & $365(7.6)$ & $1296(7.1)$ \\
Hyperparathyroidism, no. (\%) & $17(0.3)$ & $61(0.3)$ \\
Current smokers, no. (\%) & $765(16.1)$ & $3371(18.8)$ \\
Oral corticosteroid therapy, ${ }^{\text {a }}$ no. $(\%)$ & $522(10.8)$ & $911(5.0)$ \\
Injectable corticosteroid therapy, ${ }^{a}$ no. $(\%)$ & $43(0.9)$ & $32(0.2)$ \\
Statin therapy, ${ }^{a}$ no. $(\%)$ & $326(20.7)$ & $1240(20.6)$ \\
Current fluoroquinolone exposure, no. $(\%)$ & $111(2.3)$ & $236(1.3)$ \\
Current co-amoxiclav exposure, no. (\%) & $98(2.0)$ & $314(1.7)$ \\
Prior history of tendon rupture, no. (\%) & $133(2.8)$ & $114(0.6)$ \\
\hline
\end{tabular}

${ }^{a}$ At least one prescription within 90 days of the index date. Current fluoroquinolone exposure $=$ prescription within 30 days of the index date

were well matched on age, sex and duration of follow-up. The most common types of tendon rupture were: Achilles tendon (32.4\%), biceps tendon (27.5\%), shoulder tendons (16.6\%), hand/wrist tendons (7.4\%), hamstring/ quadriceps/patellar tendons $(7.0 \%)$, foot/ankle tendons $(0.8 \%)$ and triceps tendon $(0.5 \%)$. Unspecified tendon ruptures accounted for $8.3 \%$ of all cases. The mean duration of cumulative fluoroquinolone exposure was 10.6 days (standard deviation, SD 8.9 days) and for co-amoxiclav was 8.6 days (SD 7.4 days).

\subsection{Risk of Tendon Rupture with Fluoroquinolone Exposure}

The relative incidence of any tendon rupture was significantly elevated with current systemic fluoroquinolone exposure (adjusted IRR [aIRR] 1.61, 95\% CI 1.25-2.09). When stratified by type of tendon rupture, current fluoroquinolone exposure was associated with a significantly elevated rate of Achilles tendon rupture (aIRR 3.14, 95\% CI 2.11-4.65) but not biceps tendon rupture (aIRR 1.07, 95\% CI 0.61-1.89) or other tendon ruptures (aIRR $0.82,95 \%$ CI $0.50-1.35$ ) (Table 2). In contrast, the relative incidence of tendon ruptures was not significantly elevated with current co-amoxiclav exposure (Table 2).

Increasing cumulative days of systemic fluoroquinolone exposure was significantly associated with increased risk of Achilles tendon rupture (IRR 1.06, 95\% CI 1.03-1.09). In contrast, no significant association was observed with cumulative co-amoxiclav exposure (Online Supplementary Table S2). The relative incidence of Achilles tendon rupture was significantly increased with systemic fluoroquinolone exposure within 1-30 days (aIRR 2.97, 95\% CI 1.98-4.43) and 31-60 days (aIRR 2.11, 95\% CI 1.30-3.41) of the index date but not with systemic fluoroquinolone exposure occurring further in the past (aIRR $0.69,95 \%$ CI $0.41-1.16$ for $61-90$ days and aIRR $1.23,95 \%$ CI $0.88-1.71$ for $91-180$ days prior to the index date).

\subsection{Relationship with Other Risk Factors}

The adjusted relative incidence of tendon rupture was significantly elevated in people with a history of prior tendon rupture, in those with oral corticosteroid therapy, and with

Table 2 Incidence rate ratios for the association between tendon rupture and current systemic fluoroquinolone and co-amoxiclav exposure

\begin{tabular}{|c|c|c|c|c|c|}
\hline Tendon rupture & Exposed cases/total & Exposed controls/total & Crude IRR & Adjusted IRR & Adjusted $p$ value \\
\hline \multicolumn{6}{|l|}{ Any tendon rupture } \\
\hline Fluoroquinolones & $111 / 4836$ & $236 / 18,356$ & $1.79(1.41-2.27)$ & $1.61(1.25-2.09)$ & $<0.001$ \\
\hline Co-amoxiclav & $98 / 4836$ & $314 / 18,356$ & $1.15(0.90-1.45)$ & $1.02(0.79-1.31)$ & 0.900 \\
\hline \multicolumn{6}{|c|}{ Achilles tendon rupture } \\
\hline Fluoroquinolones & $67 / 1577$ & $82 / 6007$ & $3.50(2.45-5.02)$ & $3.14(2.11-4.65)$ & $<0.001$ \\
\hline Co-amoxiclav & $38 / 1577$ & $114 / 6007$ & $1.19(0.81-1.77)$ & $1.00(0.64-1.57)$ & 0.989 \\
\hline \multicolumn{6}{|c|}{ Biceps tendon rupture } \\
\hline Fluoroquinolones & $20 / 1316$ & $62 / 4946$ & $1.19(0.71-2.00)$ & $1.07(0.61-1.89)$ & 0.804 \\
\hline Co-amoxiclav & $23 / 1316$ & $74 / 4946$ & $1.16(0.72-1.88)$ & $1.01(0.61-1.66)$ & 0.978 \\
\hline \multicolumn{6}{|c|}{ Other tendon rupture } \\
\hline Fluoroquinolones & $24 / 1943$ & $92 / 7403$ & $0.94(0.59-1.50)$ & $0.82(0.50-1.35)$ & 0.439 \\
\hline Co-amoxiclav & $37 / 1943$ & $126 / 7403$ & $1.09(0.75-1.60)$ & $1.01(0.68-1.50)$ & 0.946 \\
\hline
\end{tabular}

Model adjusted for exact age, body mass index, Charlson co-morbidity score, hypothyroidism, hyperparathyroidism, smoking status, oral and injectable corticosteroid therapy, statin therapy and prior history of tendon rupture

$I R R$ incidence rate ratio 
increasing BMI (Table 3). The risk of tendon rupture was not significantly associated with statin therapy or co-morbidity score, whilst smoking was associated with a reduced relative incidence of tendon rupture. A large significant interaction was observed with concomitant systemic fluoroquinolone and oral corticosteroid exposure (aIRR 6.88, 95\% CI 3.94-12.03 and aIRR 19.36, 95\% CI 7.78-48.19, for any tendon rupture and Achilles tendon rupture, respectively, Table 4).

\subsection{Absolute Risk}

Adjusted rate differences for any tendon rupture and Achilles tendon rupture are presented overall and for age and sex (Table 5). Fluoroquinolone exposure caused an estimated 2.9 tendon ruptures and 2.1 Achilles tendon ruptures per 10,000 patients per year and was greatest in patients aged 60 years and over. Concomitant oral corticosteroid exposure had a large impact on absolute risk, with the highest rates of tendon rupture associated with concomitant fluoroquinolone and corticosteroid exposure in males and in patients aged 60 years and over.

\subsection{Sensitivity Analyses}

The relative incidence was elevated using 60-day and 90-day risk windows but was smaller in size with increasing riskwindow duration, whilst the results of all other sensitivity analyse were similar to those in the main analysis (Online Supplementary Table S3).

\section{Discussion}

Fluoroquinolone exposure significantly increased the risk of tendon rupture, which appeared to last up to 60 days following exposure for Achilles tendon rupture. Risk was also estimated to increase by $\sim 6 \%$ with each additional day exposed

Table 3 Association with any tendon rupture and Achilles tendon rupture and other potentially confounding variables and co-morbidities

\begin{tabular}{|c|c|c|c|c|}
\hline \multirow[t]{2}{*}{ Variable } & \multicolumn{2}{|l|}{ Any tendon rupture } & \multicolumn{2}{|c|}{ Achilles tendon rupture } \\
\hline & Adjusted IRR & Adjusted $p$ value & Adjusted IRR & Adjusted $p$ value \\
\hline Prior tendon rupture & $4.60(3.51-6.04)$ & $<0.001$ & $7.48(4.53-12.35)$ & $<0.001$ \\
\hline Oral corticosteroid & $2.25(1.99-2.54)$ & $<0.001$ & $3.60(2.89-4.48)$ & $<0.001$ \\
\hline Injectable corticosteroid & $5.47(3.36-8.91)$ & $<0.001$ & $1.82(0.40-8.29)$ & 0.435 \\
\hline Fluoroquinolone & $1.61(1.25-2.09)$ & $<0.001$ & $3.14(2.11-4.65)$ & $<0.001$ \\
\hline Co-amoxiclav & $1.02(0.79-1.31)$ & 0.900 & $1.00(0.64-1.57)$ & 0.989 \\
\hline Body mass index & $1.01(1.01-1.02)$ & $<0.001$ & $1.01(1.00-1.03)$ & 0.008 \\
\hline Statin therapy & $1.06(0.98-1.16)$ & 0.129 & $0.96(0.81-1.14)$ & 0.650 \\
\hline Hypothyroidism & $1.03(0.90-1.17)$ & 0.693 & $0.91(0.70-1.18)$ & 0.469 \\
\hline Hyperthyroidism & $0.89(0.51-1.55)$ & 0.676 & $1.08(0.41-2.89)$ & 0.873 \\
\hline Charlson co-morbidity & $0.99(0.97-1.01)$ & 0.279 & $1.00(0.96-1.05)$ & 0.975 \\
\hline Connective tissue disease & $2.03(1.66-2.49)$ & $<0.001$ & $1.02(0.63-1.65)$ & 0.942 \\
\hline COPD & $1.07(0.99-1.17)$ & 0.096 & $1.24(1.08-1.44)$ & 0.003 \\
\hline Cancer & $0.97(0.88-1.06)$ & 0.473 & $0.98(0.81-1.19)$ & 0.854 \\
\hline Chronic kidney disease & $0.96(0.86-1.07)$ & 0.452 & $1.02(0.82-1.28)$ & 0.865 \\
\hline Dementia & $0.47(0.34-0.66)$ & $<0.001$ & $0.34(0.14-0.79)$ & $<0.001$ \\
\hline Diabetes mellitus & $1.01(0.91-1.11)$ & 0.854 & $1.03(0.85-1.25)$ & 0.758 \\
\hline Heart failure & $0.85(0.71-1.01)$ & 0.062 & $0.87(0.64-1.25)$ & 0.459 \\
\hline Liver disease & $0.87(0.56-1.34)$ & 0.521 & $1.04(0.49-2.23)$ & 0.916 \\
\hline Myocardial infarction & $1.07(0.92-1.23)$ & 0.404 & $0.96(0.71-1.30)$ & 0.796 \\
\hline Peptic ulcer disease & $1.09(0.94-1.27)$ & 0.263 & $1.01(0.76-1.36)$ & 0.921 \\
\hline Peripheral vascular disease & $0.90(0.65-1.25)$ & 0.543 & $0.85(0.46-1.57)$ & 0.606 \\
\hline Stroke & $1.04(0.90-1.21)$ & 0.579 & $1.03(0.75-1.41)$ & 0.878 \\
\hline \multicolumn{5}{|l|}{ Smoking } \\
\hline Ex-smoker & $1.04(0.96-1.12)$ & 0.360 & $0.85(0.74-0.98)$ & 0.029 \\
\hline Current smoker & $0.85(0.78-0.94)$ & 0.001 & $0.72(0.61-0.85)$ & $<0.001$ \\
\hline
\end{tabular}

Variables included separately in the model without interactions

$I R R$ incident rate ratio, $C O P D$ chronic obstructive pulmonary disease 
Table 4 Incidence rate ratios for the interaction between tendon rupture and current systemic fluoroquinolone and oral corticosteroid exposure

\begin{tabular}{|c|c|c|c|c|}
\hline Tendon rupture & Exposed cases/total & Exposed controls/total & Adjusted IRR & Adjusted $p$ value \\
\hline \multicolumn{5}{|l|}{ Any tendon rupture } \\
\hline Oral corticosteroid alone & $287 / 4836$ & $443 / 18,356$ & $2.58(2.19-3.03)$ & $<0.001$ \\
\hline $\begin{array}{l}\text { Oral corticosteroid plus fluoro- } \\
\text { quinolone exposure }\end{array}$ & $36 / 4836$ & $20 / 18,356$ & $6.88(3.93-12.03)$ & $<0.001$ \\
\hline \multicolumn{5}{|l|}{ Achilles tendon rupture } \\
\hline Oral corticosteroid alone & $148 / 1557$ & $180 / 6007$ & $4.59(3.43-6.14)$ & $<0.001$ \\
\hline $\begin{array}{l}\text { Oral corticosteroid plus fluoro- } \\
\text { quinolone exposure }\end{array}$ & $37 / 1557$ & $10 / 6007$ & $19.36(7.78-48.19)$ & $<0.001$ \\
\hline
\end{tabular}

Adjusted for exact age, body mass index, Charlson co-morbidity score, hypothyroidism, hyperthyroidism, injectable corticosteroid therapy, smoking status, statin therapy and prior history of tendon rupture

$I R R$ incidence rate ratio

Table 5 Adjusted rate differences in tendon rupture with fluoroquinolone exposure and concomitant oral corticosteroid exposure (per 10,000 patients)

\begin{tabular}{lccccc}
\hline Variable & \multicolumn{2}{l}{ Any tendon } & & \multicolumn{2}{l}{ Achilles tendon } \\
& ARD & $95 \%$ CI & & ARD & $95 \%$ CI \\
\hline Fluoroquinolone exposure & & & & \\
Overall & 2.9 & $2.2-3.7$ & & 2.1 & $1.6-2.7$ \\
Males & 2.3 & $1.8-3.0$ & & 1.6 & $1.3-2.1$ \\
Females & 3.1 & $2.4-4.0$ & & 2.1 & $1.6-2.7$ \\
Age $<60$ years & 1.4 & $1.0-1.8$ & & 1.2 & $0.9-1.6$ \\
Age $\geq 60$ years & 5.6 & $4.3-7.2$ & & 3.0 & $2.3-3.9$ \\
Fluoroquinolone plus oral & & & & \\
$\quad$ corticosteroid exposure & & & & \\
Overall & 10.9 & $8.3-14.0$ & & 4.9 & $3.7-6.2$ \\
Males & 14.6 & $11.2-18.8$ & & 5.5 & $4.2-7.1$ \\
Females & 7.9 & $6.0-10.1$ & & 3.9 & $3.0-5.0$ \\
Age $<60$ years & 6.0 & $4.6-7.7$ & & 2.1 & $1.6-2.7$ \\
Age $\geq 60$ years & 19.6 & $15.0-25.2$ & & 6.6 & $5.0-8.4$ \\
\hline
\end{tabular}

$A D R$ adjusted rate difference, the additional number of expected events per 10,000 patients per year

to fluoroquinolones within the current risk window and was increased markedly with concomitant exposure to oral corticosteroids. In contrast, co-amoxiclav exposure, which was chosen as a negative control, was not associated with an increased relative risk of any tendon rupture. Absolute risk of tendon rupture from fluoroquinolone exposure was greater than currently highlighted by product information and varied markedly according to age and concomitant exposure to oral corticosteroids.

Observational studies have previously investigated the risk of tendon rupture with fluoroquinolones with results varying in the size and precision of estimates. One case-control study consisting of only 38 cases of Achilles tendon rupture reported a relative risk with current exposure to fluoroquinolone antibiotics of 1.9 (95\% CI 1.3-2.6), whilst a larger case-control study from Italy reported an $30 \%$ increased risk of any tendon rupture and a larger but less precise risk of Achilles tendon rupture (OR 4.1, 95\% CI 1.8-9.6 for Achilles tendon rupture) [16, 18]. Meanwhile, a small cohort study from Denmark has reported an agestandardized incidence ratio of Achilles tendon rupture of 3.1 (95\% CI 1.0-7.3) within 90 days of incident fluoroquinolone exposure whilst a further case-control study reported a larger risk of Achilles tendon rupture (odds ratio (OR) 5.3, 95\% CI 1.8-15.2) [17, 19]. However, these studies did not include an antibiotic-treated comparator in their design to help assess the presence of residual confounding.

One case-crossover study, whereby the patient acts as their own control, reported a smaller association with Achilles tendon rupture (OR 2.0, 95\% CI 1.2-3.3) and used a composite of nitrofurantoin, amoxicillin and trimethoprim exposure as a negative control with no significant association being observed [10]. This comparator group consisted of medications weighted towards the treatment of milder urinary tract infections. In contrast, a cohort study from Canada reporting an increased risk of tendon rupture with fluoroquinolones did use an active comparator of amoxicillin exposure that was also significantly associated with tendon rupture, suggesting the presence of residual confounding can affect the validity and size of the relative risk [20]. We used co-amoxiclav as a negative control because in the UK coamoxiclav is a similar broader-spectrum antibiotic reserved for more severe types of infection and may be less subject to unmeasured confounding compared to amoxicillin, nitrofurantoin and trimethoprim. Similar to fluoroquinolones, co-amoxiclav is also associated with an increased risk of Clostridium difficile infection, which has resulted in similar changes to UK antibiotic prescribing guidelines during the study period.

Our study extends knowledge by demonstrating that risk appears to be present up to 60 days following treatment 
and increases by $\sim 6 \%$ with each day of current exposure. People with prior tendon rupture, co-morbidity, those with increasing BMI and those prescribed oral corticosteroids were at increased risk of tendon rupture, with a significant interaction detected between concomitant fluoroquinolone and oral corticosteroid therapy. This interaction has previously been reported but with variable precision, with an OR ranging from 9.1 to $43.2[10,16,19]$. Information on absolute risk is limited. Van der Linden et al. [16] report an excess absolute risk of Achilles tendon rupture of 3.2 cases per 1000 patient years, although the method used to calculate this was not reported. In a related study, van der Linden et al. [17] report the incidence of Achilles tendon rupture in people aged 60 years and over as a populationattributable risk, estimating that $2.2 \%$ of Achilles tendon rupture in patients aged $60-79$ years and $6.3 \%$ in patients aged 80 years and over are attributable to fluoroquinolone exposure. This absolute risk estimate may be dependent on the prevalence of exposure in the population though. Although low, we found that absolute risk in this population may be greater than that listed in the product information for fluoroquinolones (which states less than 1 in 10,000 patients). Although we estimate a populationaverage adjusted rate difference (akin to excess risk) of $\sim 2.9$ tendon ruptures per 10,000 person years, absolute risk was greater in certain subgroups, significantly varying by age, concomitant corticosteroid exposure and, to a lesser extent, gender. Lastly, statin therapy has previously been linked to tendon disorders using spontaneous reports and pharmacovigilance databases [30]. Our study found no significant increased risk with statin use, in keeping with three other recent observational studies [31-33].

Our study has strengths and limitations. We matched on general practice so that controls were more likely to have similar socio-economic deprivation status and health-care physician prescribing behavior, helping to reduce confounding by indication. Only small numbers of cases were included unmatched on general practice, with sensitivity analysis demonstrating this had negligible influence on the observed results. Despite adjustment for multiple confounders, risk of residual unmeasured confounding remains possible, which may include channeling bias, although evaluating the risk of tendon rupture with co-amoxiclav exposure consistently showed no statistically significant association. Very few traumatic tendon ruptures were explicitly recorded and it is not possible to determine what proportion of other events may have been associated with trauma, if any. Antibiotic exposures were identified through prescriptions issued within general practice. Our study focuses on tendon rupture only and further investigation on the risk of tendinitis may be useful for clinical decision making. Similarly, we focus on fluoroquinolone as a class and risk associated with individual fluoroquinolone products requires further evaluation.
Antibiotic stewardship aimed at reducing fluoroquinolone exposure may prevent unnecessary harm but this may not be possible or appropriate for all patients. Despite the relatively large increase in risk associated with concomitant exposure to systemic fluoroquinolones and corticosteroids, relatively little attention is given to this interaction among clinical guideline recommendations. This may have implications for special patient groups such as in the management of asthma, chronic obstructive pulmonary disease or bronchiectasis exacerbations, which may require concomitant use of both antibiotics and oral corticosteroids regimens, for example, and commonly have other risk factors such as co-morbidity [34-36].

This study was conducted to provide further information to quantify the risk of tendon ruptures associated with fluoroquinolone antibiotics to support a review of safety information at the EMA PRAC in order to aid regulatory decision making for the use of fluoroquinolones across Europe. In this regard, tendon rupture is only one potential adverse effect of fluoroquinolone exposure, with other potential adverse effects associated with peripheral neuropathy and aortic aneurysm having been reported [20, 37-40].

In conclusion, the risk of tendon rupture associated with fluoroquinolone therapy appears to depend on timing, dose and concomitant exposure to corticosteroids, and affects elderly patients the greatest in absolute terms; patients and healthcare professionals should be informed of these risks.

\section{Compliance with Ethical Standards}

Funding No source of funding was used for this study.

Conflict of interest The authors have no conflicts of interest to declare.

Ethical approval Approval to conduct the studies using anonymised data was granted by the Scientific Review Committees of The Health Improvement Network (Protocol number 17THIN087).

Disclaimer The views expressed in this article are the personal views of the author(s) and may not be not be understood or quoted as reflecting the views of the EMA or one of its committees or working parties.

Author Contributions All authors were involved in the study design and data collection. DM performed the analysis and is guarantor for the study. All authors contributed to the interpretation of results and writing the manuscript, and approved the final draft.

Open Access This article is distributed under the terms of the Creative Commons Attribution-NonCommercial 4.0 International License (http://creativecommons.org/licenses/by-nc/4.0/), which permits any noncommercial use, duplication, adaptation, distribution and reproduction in any medium or format, as long as you give appropriate credit to the original author(s) and the source, a link is provided to the Creative Commons license and any changes made are indicated. 


\section{References}

1. Morales DR, Slattery J, Pinheiro L, Kurz X, Hedenmalm K. Indications for systemic fluoroquinolone therapy in Europe and prevalence of primary-care prescribing in France, Germany and the UK: descriptive population-based study. Clin Drug Investig. 2018;38(10):927-33.

2. Ciproxin $500 \mathrm{mg}$ tablet summary of product characteristics. https://www.medicines.org.uk/emc/product/6153/smpc. Accessed 06 July 2018

3. Khaliq Y, Zhanel GG. Fluoroquinolone-associated tendinopathy: a critical review of the literature. Clin Infect Dis. 2003;36(11):1404-10.

4. Ozaras R, Mert A, Tahan V, Uraz S, Ozaydin I, Yilmaz MH, Ozaras N. Ciprofloxacin and Achilles' tendon rupture: a causal relationship. Clin Rheumatol. 2003;22(6):500-1.

5. Khan RJ, Carey Smith RL. Surgical interventions for treating acute Achilles tendon ruptures. Cochrane Database Syst Rev. 2010. https://doi.org/10.1002/14651858.CD003674.pub4

6. Huttunen TT, Kannus P, Rolf C, Fellander-Tsai L, Mattila VM. Acute Achilles tendon ruptures: incidence of injury and surgery in Sweden between 2001 and 2012. Am J Sports Med. 2014;42:2419-23.

7. Maffulli N, Waterston SW, Squair J, Reaper J, Douglas AS. Changing incidence of Achilles tendon rupture in Scotland: a 15-year study. Clin J Sport Med. 1999;9(3):157-60.

8. Kelly MP, Perkinson SG, Ablove RH, Tueting JL. Distal biceps tendon ruptures: an epidemiological analysis using a large population database. Am J Sports Med. 2015;43(8):2012-7.

9. Tsai WC, Hsu CC, Chen CP, et al. Ciprofloxacin up-regulates tendon cells to express matrix metalloproteinase-2 with degradation of type I collagen. J Orthop Res. 2011;29:67-73.

10. Wise BL, Peloquin C, Choi H, Lane NE, Zhang Y. Impact of age, sex, obesity, and steroid use on quinolone-associated tendon disorders. Am J Med. 2012;125(12):1228.e23-8.

11. Józsa L, Kvist M, Bálint BJ, Reffy A, Järvinen M, Lehto M, Barzo M. The role of recreational sport activity in Achilles tendon rupture. A clinical, pathoanatomical, and sociological study of 292 cases. Am J Sports Med. 1989;17:338-43.

12. Spoendlin J, Meier C, Jick SS, Meier CR. Achilles or biceps tendon rupture in women and men with type 2 diabetes: a population-based case-control study. J Diabetes Complicat. 2016;30:903-9.

13. Szarfman A, Chen M, Blum MD. More on fluoroquinolone antibiotics and tendon rupture. N Engl J Med. 1995;332(3):193.

14. FDA drug safety communication (online). July 2016. http:// www.fda.gov/Drugs/DrugSafety/ucm500143.htm. Accessed 17 Jan 2017.

15. European Medicines Agency. Quinolone and fluoroquinolone article 31 referral. 2017. http://www.ema.europa.eu/ema/index .jsp?curl=pages/medicines/human/referrals/Quinolones_and_ fluoroquinolones_containing_medicinal_products/human_refer ral_prac_000065.jsp\&mid=WC0b01ac05805c516f. Accessed 01 Sept 2017.

16. van der Linden PD, Sturkenboom MC, Herings RM, Leufkens HG, Stricker BH. Fluoroquinolones and risk of Achilles tendon disorders: case-control study. BMJ. 2002;324(7349):1306-7.

17. van der Linden PD, Sturkenboom MC, Herings RM, Leufkens HM, Rowlands S, Stricker BH. Increased risk of achilles tendon rupture with quinolone antibacterial use, especially in elderly patients taking oral corticosteroids. Arch Intern Med. 2003;163(15):1801-7.

18. Sode J, Obel N, Hallas J, Lassen A. Use of fluroquinolone and risk of Achilles tendon rupture: a population-based cohort study. Eur J Clin Pharmacol. 2007;63(5):499-503.
19. Corrao G, Zambon A, Bertù L, Mauri A, Paleari V, Rossi C, Venegoni M. Evidence of tendinitis provoked by fluoroquinolone treatment: a case-control study. Drug Saf. 2006;29(10):889-96.

20. Daneman N, Lu H, Redelmeier DA. Fluoroquinolones and collagen associated severe adverse events: a longitudinal cohort study. BMJ Open. 2015;5(11):e010077.

21. Seeger JD, West WA, Fife D, Noel GJ, Johnson LN, Walker AM. Achilles tendon rupture and its association with fluoroquinolone antibiotics and other potential risk factors in a managed care population. Pharmacoepidemiol Drug Saf. 2006;15:784-92.

22. Blak BT, Thompson M, Dattani H, Bourke A. Generalisability of The Health Improvement Network (THIN) database: demographics, chronic disease prevalence and mortality rates. Inform Prim Care. 2011;19(4):251-5.

23. Health and Social Care Information Centre. Read codes. http:// systems.hscic.gov.uk/data/uktc/readcodes. Accessed 1 Sept 2015.

24. Lewis JD, Schinnar R, Bilker WB, Wang X, Strom BL. Validation studies of the health improvement network (THIN) database for pharmacoepidemiology research. Pharmacoepidemiol Drug Saf. 2007;16(4):393-401.

25. Etminan M, Samii A. Pharmacoepidemiology I: a review of pharmacoepidemiologic study designs. Pharmacotherapy. 2004;24:964-9.

26. Joint Formulary Committee. British National Formulary (online) London: BMJ Group and Pharmaceutical Press. http://www.medic inescomplete.com. Accessed 16 Oct 2016.

27. Charlson ME, Pompei P, Ales KL, MacKenzie CR. A new method of classifying prognostic comorbidity in longitudinal studies: development and validation. J Chronic Dis. 1987;40:373-83.

28. Suissa S. The Quasi-cohort approach in pharmacoepidemiology: upgrading the nested case-control. Epidemiology. 2015;26:242-6.

29. van Buuren S. Multiple imputation of discrete and continuous data by fully conditional specification. Stat Methods Med Res. 2007;16:219-42.

30. Marie I, Delafenêtre H, Massy N, Thuillez C, Noblet C, Network of the French Pharmacovigilance Centers. Tendinous disorders attributed to statins: a study on ninety-six spontaneous reports in the period 1990-2005 and review of the literature. Arthritis Rheum. 2008;59(3):367-72.

31. Spoendlin J, Layton JB, Mundkur M, Meier C, Jick SS, Meier $\mathrm{CR}$. The risk of Achilles or biceps tendon rupture in new statin users: a propensity score-matched sequential cohort study. Drug Saf. 2016;39(12):1229-37.

32. Contractor T, Beri A, Gardiner JC, Tang X, Dwamena FC. Is statin use associated with tendon rupture? A population-based retrospective cohort analysis. Am J Ther. 2015;22(5):377-81.

33. Beri A, Dwamena FC, Dwamena BA. Association between statin therapy and tendon rupture: a case-control study. J Cardiovasc Pharmacol. 2009;53(5):401-4.

34. Weatherburn CJ, Guthrie B, Mercer SW, Morales DR. Comorbidities in adults with asthma: population-based cross-sectional analysis of 1.4 million adults in Scotland. Clin Exp Allergy. 2017;47(10):1246-52.

35. Morales DR, Lipworth BJ, Donnan PT, Jackson C, Guthrie B. Respiratory effect of beta-blockers in people with asthma and cardiovascular disease: population-based nested case control study. BMC Med. 2017;15(1):18.

36. Chetty U, McLean G, Morrison D, Agur K, Guthrie B, Mercer SW. Chronic obstructive pulmonary disease and comorbidities: a large cross-sectional study in primary care. Br J Gen Pract. 2017;67(658):e321-8.

37. Etminan M, Brophy JM, Samii A. Oral fluoroquinolone use and risk of peripheral neuropathy: a pharmacoepidemiologic study. Neurology. 2014;83(14):1261-3. 
38. Lee CC, Lee MG, Hsieh R, Porta L, Lee WC, Lee SH, Chang SS. Oral fluoroquinolone and the risk of aortic dissection. J Am Coll Cardiol. 2018;72(12):1369-78.

39. Pasternak B, Inghammar M, Svanström H. Fluoroquinolone use and risk of aortic aneurysm and dissection: nationwide cohort study. BMJ. 2018;8(360):k678.
40. Lee CC, Lee MT, Chen YS, Lee SH, Chen YS, Chen SC, Chang SC. Risk of aortic dissection and aortic aneurysm in patients taking oral fluoroquinolone. JAMA Intern Med. 2015;175(11):1839-47. 